\title{
Ideal magnetohydrodynamic constraints on the pedestal temperature in tokamaks
}

\author{
P B Snyder ${ }^{1}$ and H R Wilson ${ }^{2}$ \\ ${ }^{1}$ General Atomics, PO Box 85608, San Diego, CA 92186-5608, USA \\ 2 EURATOM/UKAEA Fusion Association, Culham Science Centre, Abingdon, Oxon \\ OX14 3DB, UK
}

Received 6 March 2003, in final form 6 June 2003

Published 19 August 2003

Online at stacks.iop.org/PPCF/45/1671

\begin{abstract}
The ideal magnetohydrodynamic (MHD) stability limits for the edge transport barrier (ETB) region in tokamaks are explored, concentrating in particular on the intermediate to high toroidal mode number, $n$, modes. These calculations take full account of the effect of the edge bootstrap current on the stability of both ballooning and peeling modes. Because the current plays an important role in MHD stability, the temperature and density independently influence stability and, in particular, the pressure gradient that the ETB can support. The stability calculations therefore provide limits to the achievable temperature pedestal associated with the transport barrier which are not simply pressure pedestal limits, as is often assumed. One important result is that increasing triangularity is predicted to be beneficial in providing access to a higher temperature pedestal at fixed pedestal width, at least up to triangularity $\sim 0.5$. Another significant result is that the finite $n$ corrections, which are stabilizing for ballooning modes, are important for narrow pedestal widths and permit significantly higher temperature pedestals than one would obtain using the leading order $(n=\infty)$ ballooning theory. Specific calculations for equilibria characteristic of ITER and FIRE suggest that temperature pedestals in the region of a few kiloelectronvolts should be achievable, but the precise value depends on the pedestal width, a prediction for which is beyond the scope of this paper.
\end{abstract}

\section{Introduction}

The confinement in tokamaks is widely believed to be governed by plasma turbulence which yields large transport when the temperature gradient exceeds a critical value; this leads to so-called 'stiff' transport models. For these, the overall confinement is sensitive to the value of the temperature close to the plasma edge, which serves as a boundary condition for the core transport modelling. For high confinement 'H-mode' discharges, the good confinement is achieved as a result of suppressing turbulence in the edge plasma region, leading to a 
transport barrier able to accommodate steep pressure gradients. The temperature used in the core transport modelling of such discharges is that at the inner edge of this transport barrier, and is often referred to as the pedestal temperature. Thus, the larger the pedestal temperature at a given density, the better the confinement. Previous studies using stiff transport models have generally found that the fusion power of proposed reactors scales roughly with the square of the pedestal height [1].

Two ingredients are required to determine the pedestal temperature: the width of the transport barrier and the temperature gradient it can support. While there have been many theories proposed in the literature, the mechanism for the production of the barrier width is still not completely understood, but it is unlikely to be entirely a consequence of ideal magnetohydrodynamic (MHD) stability, and is therefore beyond the scope of this paper. On the other hand, ideal MHD stability can constrain the maximum achievable gradients in the transport barrier and thus, by making assumptions about the barrier width, one can deduce values for the maximum achievable temperature pedestal.

There are two basic instabilities that govern ideal MHD in the plasma edge region. First, the high toroidal mode number, $n$, ballooning mode is driven by edge pressure gradient, and therefore imposes a direct limit on the temperature gradient that can be maintained in the transport barrier. On the other hand, for shaped plasmas, sufficient current density can stabilize the ballooning mode, providing access to higher pressure gradients: so-called 'second stability' regime. The other instability is the peeling mode [2], which is strongly related to the kink mode, but is not necessarily restricted to low $n$. The peeling mode is highly localized at the plasma edge, and is driven by the edge current density; in contrast to the ballooning mode, the peeling mode is stabilized by pressure gradient (i.e. a larger edge current density can be tolerated at higher pressure gradient). A final twist to the story is that at high pressure gradient and edge current density the ballooning and peeling modes can couple [3], leading to particularly dangerous modes driven by both current density and pressure gradient, with a relatively large radial extent, typically extending right across the transport barrier region. This coupling of the two modes prevents second stability access, and has been proposed to provide the trigger for Type I edge localized modes (ELMs) [3,4]. For higher plasma shaping, the modes become de-coupled, and second stability access is again possible. Schematic stability diagrams for weak and strong shaping are shown in figure 1.

Thus, the picture of edge stability is somewhat complicated. Indeed, it is further complicated by the strong dependence of the edge current density on the edge pressure gradient and plasma collisionality. Typically, the current density in the transport barrier region is dominated by the bootstrap current [5], which is proportional to the pressure gradient, but also suppressed in higher collisionality plasmas. Thus, consider what might happen as one raises the temperature pedestal height, keeping all other parameters (in particular, the barrier width)
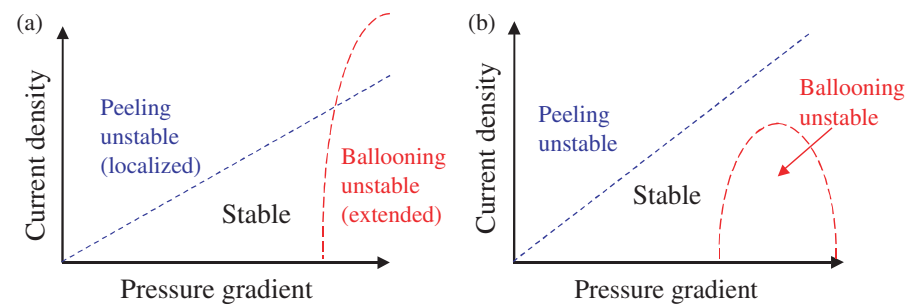

Figure 1. Schematic high $n$-MHD stability diagrams for the $(a)$ weak shaping and $(b)$ strong shaping, showing access to the second stability regime. 
constant. First, the edge pressure gradient will rise, so that the plasma edge gradients will begin to approach the ballooning limit. On the other hand, the increase in pressure gradient is beneficial for peeling modes. In addition, the higher temperature will result in a reduction of the collisionality so that, combined with the increase in edge gradient, the bootstrap current will increase: this will tend to destabilize peeling modes, but be beneficial in providing the possibility of second stability access for the ballooning modes. In short, there are a number of competing effects, and the edge stability properties of edge transport barriers (ETBs) in tokamaks are not straight forward to unravel.

In this paper, we employ the ELITE code [6], which has been designed specifically to explore the edge MHD properties of tokamak plasmas. In section 2, we describe the family of equilibria that we have generated to form the basis of our stability study, which are based on those of the ITER-FEAT baseline scenario (note that the ITER-FEAT design has recently been renamed ITER, and we will refer to it simply as ITER in the remainder of this paper). Then in section 3, we show how the edge MHD stability constraints do indeed provide a limit for the maximum achievable pedestal temperature, and explore some of the basic properties. We illustrate some of the typical mode structures, and postulate ELM characteristics that might be expected to arise from these. In section 4, we perform a more detailed study of the various dependencies of the maximum temperature pedestal on plasma parameters such as density and triangularity for equilibria representative of both FIRE and ITER. Finally, in section 5, we draw some conclusions and discuss some of the consequences of our results.

\section{Equilibrium properties}

In this section, we describe how the basic set of equilibria are generated for the studies described in section 3. We employ the SCENE code [7] to solve the Grad Shafranov equation, fixing the parameters to be similar to those of ITER as shown in table 1 (note, we do not include the effects of the separatrix here, for simplicity). In addition, we must define profiles for the pressure and current. The pressure profile is determined from the chosen forms for the density and temperature profiles, which are:

$f(\psi)=f_{\mathrm{a}}+\left(f_{\text {ped }}-f_{\mathrm{a}}\right) \tanh \lambda\left(\frac{\psi_{\mathrm{a}}-\psi}{\psi_{\mathrm{a}}-\psi_{0}}\right)+\left(f_{0}-f_{\text {ped }}\right)\left(\frac{\psi_{\mathrm{a}}-\psi}{\psi_{\mathrm{a}}-\psi_{0}}\right)^{\mu}$

where $f$ represents either the density or temperature profiles as a function of the poloidal magnetic flux, $\psi$. The value at the magnetic axis is represented by $f_{0}$, the pedestal value by

Table 1. Parameters of the base-line equilibrium used in sections 2 and 3, representative of ITER.

\begin{tabular}{ll}
\hline Parameter & Value \\
\hline Major, minor radius & $6.2 \mathrm{~m}, 2.0 \mathrm{~m}$ \\
Triangularity & $0.33,0.5$ \\
Elongation & 1.7 \\
Plasma current & $15 \mathrm{MA}$ \\
Magnetic field & $5.3 \mathrm{~T}$ \\
Temperature pedestal width & $7.0 \mathrm{~cm}$ \\
Line average density & $1 \times 10^{20} \mathrm{~m}^{-3}$ \\
Edge density & $3 \times 10^{19} \mathrm{~m}^{-3}$ \\
Central temperature & $24 \mathrm{keV}$ \\
Edge temperature & $250 \mathrm{eV}$ \\
Pedestal temperature & $4.0 \mathrm{keV}$ \\
$\beta_{\mathrm{N}}$ & 1.5 \\
\hline
\end{tabular}


$f_{\text {ped }}$ and the edge value by $f_{\mathrm{a}}$. We take a very flat density profile, so that $n_{\text {ped }}=n_{0}, \mu=0$ and we fix the pedestal width parameter $\lambda=10$; the edge density, $n_{\mathrm{a}}=0.3 n_{0}$. For the temperature profile, we allow for some peaking in the core and set $\mu=2$. Our first study, to be presented in the following section, explores stability as a function of normalized pressure, $\beta_{\mathrm{N}}$, and pedestal temperature, $T_{\text {ped }}$. For this, we fix the ratio of $T_{0}: T_{\text {ped }}: T_{\mathrm{a}}=24: 4: 0.25$; varying $n_{0}$ and $T_{0}$ then allows us to scan $\beta_{\mathrm{N}}$ and $T_{\mathrm{ped}}$.

It is worth commenting that in general the density and temperature profiles will not be of the precise form assumed in equation (1), but rather be the result of transport process and sources and sinks of particles and heat. In the absence of a complete transport analysis, which is beyond the scope of this paper, we propose to use the form given in equation (1), which we believe to be representative of the true experimental situation. This provides us with a suitable basis to explore the general trends in the ideal MHD stability of the plasma edge region as the pedestal parameters are varied. Different assumptions about the pedestal profile shapes may lead to slightly different quantitative predictions, but such effects are generally expected to be small.

Turning now to the current profile $[8,9]$, we use a combination of bootstrap and Ohmic contributions, as predicted by neoclassical theory. We assume that the current profile is fully relaxed with a loop voltage independent of the minor radius (the loop voltage is constrained by the total plasma current). Typically, we find that the current density in the edge region is then dominated by the bootstrap current: this increases with $\beta_{\mathrm{N}}$ due to the increase in edge pressure gradient, and also increases with $T_{\text {ped }}$ due to the decrease in edge collisionality.

\section{Edge MHD stability}

A set of equilibria is generated according to the prescription described in the previous section. By varying $n_{0}$ and $T_{0}$ we can thus scan a range of edge current density and $\beta_{\mathrm{N}}$. This set of equilibria is then tested for stability using the ideal MHD code, ELITE [6], which has been specifically developed to explore edge plasma stability to modes with intermediate to large toroidal mode number, $n$. Comparison with low $n$ stability codes has previously shown that the expansion in $n^{-1}$ employed in the ELITE code is valid down to $n \sim 5,[6,13]$, so in this study we scan a range of toroidal mode number $5<n<31$ for each equilibrium. The resulting stability diagram is shown in figure 2 for the higher triangularity, $\delta=0.5$ case of table 1 . Here, $\langle J\rangle$ is the total plasma current divided by the poloidal cross-sectional area and $J_{\|}$is a flux surface average of the parallel current density at the plasma-vacuum interface (weighted so that the Pfirsch-Schlüter current has zero contribution). An interesting feature of the stability diagram is that the stability boundary limiting $J_{\|}$(essentially due to a peeling mode) is not a smooth function. This is because peeling mode stability for any particular mode

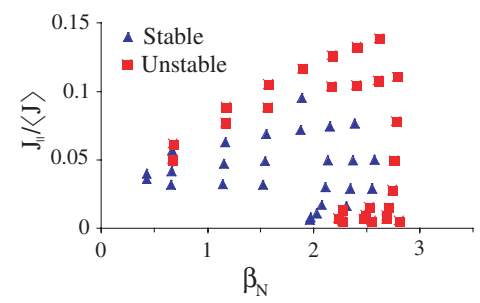

Figure 2. Edge stability diagram for an ITER-like equilibrium with triangularity $\delta=0.5$; triangles correspond to stable equilibria, while squares are unstable equilibria. 

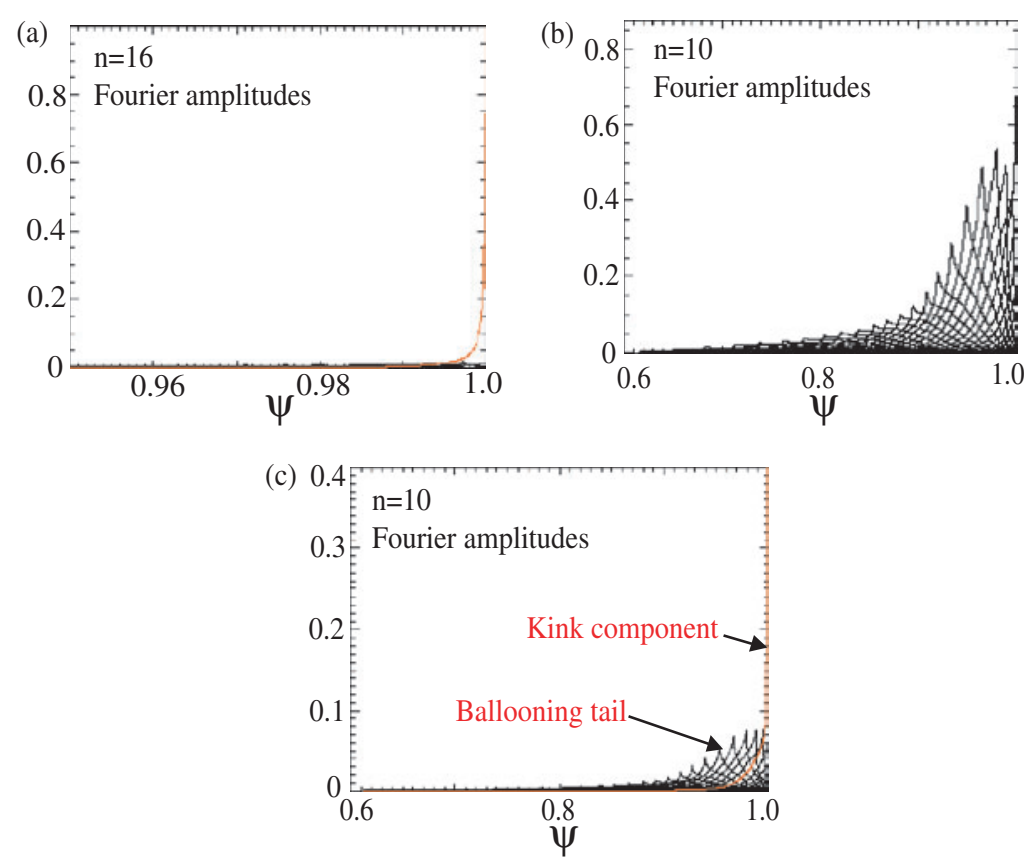

Figure 3. Mode structure for $(a)$ a peeling mode $\left(\beta_{\mathrm{N}}=1.56, J_{\|} /\langle J\rangle=0.088\right),(b)$ a $n=10$ ballooning mode $\left(\beta_{\mathrm{N}}=2.76, J_{\|} /\langle J\rangle=0.050\right)$, and $(c)$ a $n=10$ peeling-ballooning mode $\left(\beta_{\mathrm{N}}=2.40, J_{\|} /\langle J\rangle=0.104\right)$. $\psi_{\mathrm{N}}$ is the poloidal flux normalized so that $\psi_{\mathrm{N}}=0$ at the magnetic axis and $\psi_{\mathrm{N}}=1$ at the boundary.

number, $n$, is rather sensitive to the distance of the external rational surface from the plasma surface, which changes as the equilibrium (in particular, the edge safety factor) is modified. By considering a range of $n$, and choosing the most unstable for each equilibrium, the stability curve is less sensitive to the edge $q$, but some dependence remains; this is the reason for the 'raggedness' in the stability boundary seen in the figure 2.

At the lower $\beta_{\mathrm{N}}$ values, the most unstable $n$ values are those which have a vacuum rational safety factor, $q$, value close to the plasma, and are dominated by a single Fourier mode, as expected for peeling type modes: they are generally extremely edge localized (within the last $1 \%$ of the poloidal flux) (see figure 3(a)). The maximum achievable $\beta_{\mathrm{N}}=\beta[\%] a[\mathrm{~m}] B[\mathrm{~T}] / I_{\mathrm{p}}[\mathrm{MA}]$ is limited by ballooning type modes, with the highest $n$ modes being the most unstable. These modes tend to be rather radially extended, with a width comparable to the pedestal width (see figure $3(b)$ ). At high $\beta_{\mathrm{N}}$, high current density the modes are characteristic of peelingballooning modes, with a dominant Fourier component resonant just in the vacuum, as for the peeling mode, but coupled to a ballooning 'tail' penetrating well into the pedestal region (see figure $3(c)$ ). The plots in figure 3 show the radial structure of the amplitudes of the poloidal Fourier harmonics, each of which peaks at the corresponding mode rational surface, i.e. where its poloidal mode number $m=n q$. The classic characteristic of a ballooning mode is shown in figure 3(b), where each of these Fourier amplitudes all have the same shape, and are strongly localized around their respective rational surfaces. Infinite $n$ ballooning analysis shows that the cluster of points at low current density, high $\beta_{\mathrm{N}}$ in figure 3 is unstable, and indeed ELITE predicts the highest toroidal mode numbers to be the most unstable in this region, as expected from ballooning theory [10]. As an example, figure 4 shows the $s-\alpha$ diagram (obtained from the $n \rightarrow \infty$ ballooning theory) corresponding to the equilibrium that results in the ballooning 

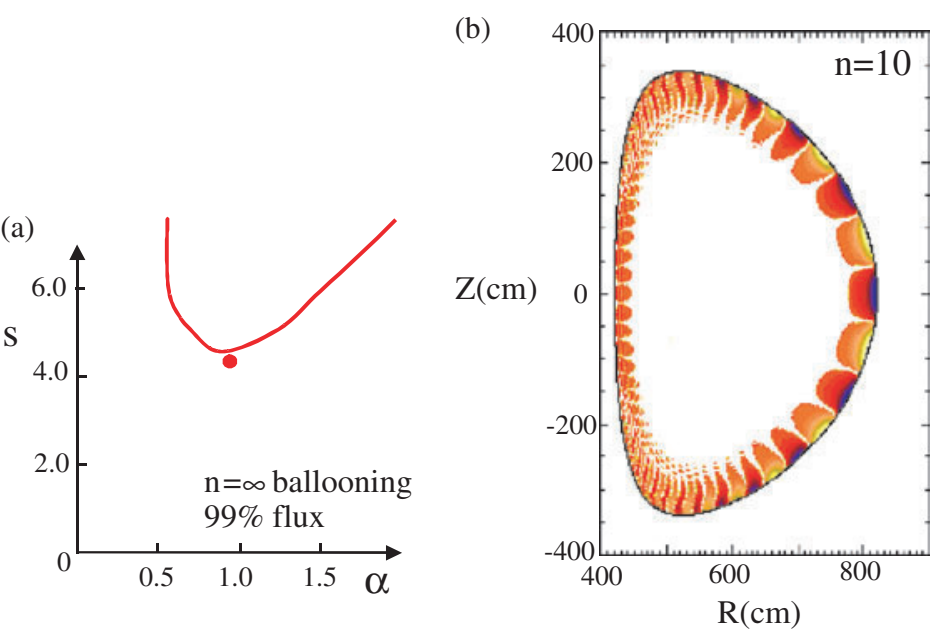

Figure 4. (a) The $s-\alpha$ diagram for the $99 \%$ flux surface, showing the proximity of the equilibrium (cross) to the marginal stability curve. (b) Also shown is the ballooning mode structure in the poloidal cross-section predicted by ELITE for this same equilibrium.

mode of figure $3(b)$ : note that it is indeed close to the predicted marginal stability curve for this mode. Also shown in figure 4 is the structure obtained from ELITE for this same mode in the poloidal cross-section. Note its amplitude peaks on the outboard side, characteristic of a ballooning mode. The $s-\alpha$ curve illustrates an interesting point: while the $n=\infty$ ballooning stability predicts that for this current density (or equivalently magnetic shear, $s$ ) the pressure gradient (parameterized by $\alpha$ in figure $4(a)$ ) can be increased arbitrarily, we find from ELITE that in fact the pressure gradient is ultimately limited by intermediate $n$ peeling- (or kink-) ballooning modes $(n \sim 6-8)$.

In general, the edge current is not well diagnosed in a tokamak, and therefore it is difficult to assess the implications of stability diagrams such as that of figure 2. However, for a given magnetic geometry there is a one-to-one, monotonic relationship between the plasma current density and edge temperature: higher temperature (at fixed pressure) leads to lower collisionality (increasing the bootstrap current) and lower resistivity (increasing the Ohmic current). Let us assume that the plasma edge is in a steady state equilibrium, so that the edge current density is given by a combination of the bootstrap current and Ohmic current (driven by a steady, externally applied loop voltage). We then find that the stability diagram can be cast in the form shown in figure 5 for (a) $\delta=0.3$ and (b) $\delta=0.5$. It should be noted that there are two possibilities for the maximum achievable temperature pedestal: either through the limit imposed on the pressure gradient by ballooning mode stability (the $\beta_{\mathrm{N}}$ limit), or a direct $T_{\text {ped }}$ limit due to its effect on the collisionality (which influences the bootstrap current and therefore the peeling mode stability). Note that a somewhat higher pedestal temperature is permitted at higher triangularity. It is also interesting to note that there is increased access to second stability in the higher triangularity case; this can be seen by the fact that higher $\beta_{\mathrm{N}}$ can be achieved at higher pedestal temperature (corresponding to higher edge current density). Note, however, as discussed earlier, there is never complete second stability access (which would be predicted by the infinite $n$ ballooning theory) due to intermediate $n$ modes being destabilized (i.e. $n \sim 6-8$ ).

In general, because of the effect of ELMs and the different timescales associated with pressure and current diffusion, the plasma edge is not in steady state in realistic tokamak 

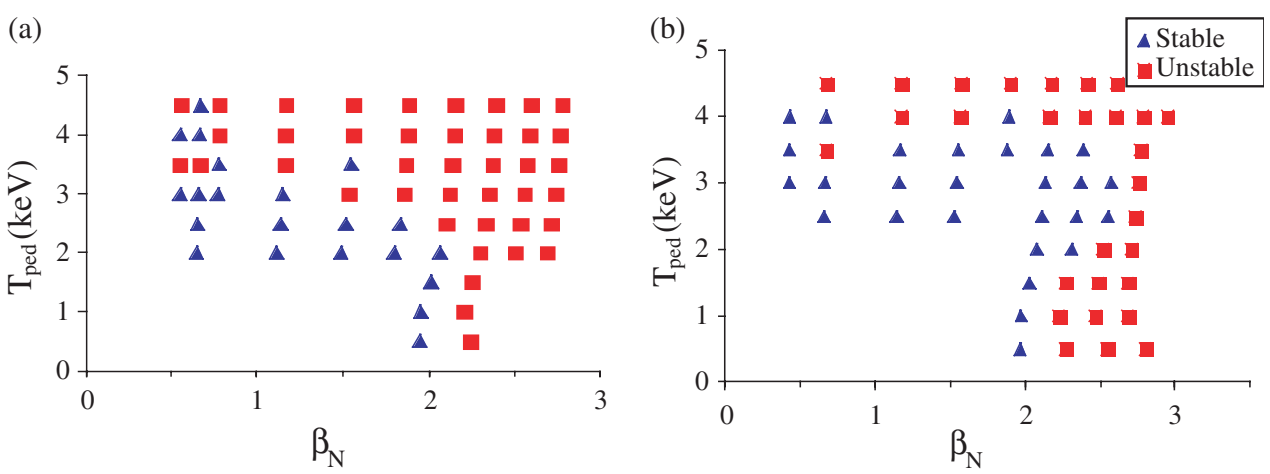

Figure 5. Stability diagrams for an ITER-like discharge for $(a) \delta=0.3$ and $(b) \delta=0.5$.

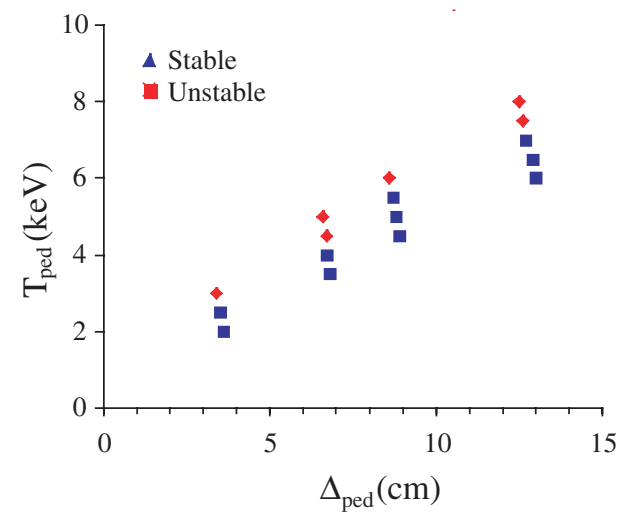

Figure 6. The stable/unstable pedestal temperature values as a function of pedestal width (and range of $6<n<30$ is scanned).

situations and it is strictly necessary to solve a set of time-dependent transport equations. However, the temperature pedestal limits shown above indicate the highest pedestals that can be expected from stability constraints (a poor transport barrier may lead to lower pedestal values, limited by edge confinement, rather than the stability). In addition, if one makes the further assumption that the larger ELMs observed in tokamaks are associated with radially extended ideal MHD instabilities (an assumption supported by previous studies, e.g. [4, 11, 12, 13]), stability diagrams like those shown in figure 5 may provide guidance for optimizing the confinement (i.e. the pedestal temperature) while avoiding the largest ELMs.

So far we have concentrated on equilibria with a fixed pedestal width $(\sim 7 \mathrm{~cm})$. As there is considerable uncertainty in predictions of this quantity for future tokamaks (in particular, those with a burning plasma), it is interesting to study stability as this is varied. Thus, in figure 6 we show the stability analyses for a range of equilibria as the pedestal width and temperature are varied, keeping other parameters close to those of the $\delta=0.5$ case of table 1 (here we choose to fix the central and edge temperatures, so that to keep $\beta_{\mathrm{N}}$ fixed, the density must be permitted to vary a little). The first point to note about this figure is that the marginally stable pedestal temperature is not simply proportional to the pedestal width: this is due largely to the increased stabilizing effects of the higher order (in $n^{-1}$ ) terms predicted by the high $n$ ballooning theory, which are larger for a narrower pedestal width. Thus, simple models based on a pressure gradient limit would tend to under-estimate the maximum achievable pedestal height at low 
values of the pedestal width. Indeed, the stabilizing effects of ion diamagnetism (not included here) may further increase the achievable pedestal temperature at more narrow widths.

\section{Pedestal stability scaling for model burning plasma equilibria}

As discussed earlier, the combined need to achieve a high pedestal for good confinement, while maintaining small or no ELMs to avoid fast erosion of plasma facing components, has emerged as a critically important issue with respect to planned next step burning plasma devices. The importance of this issue, together with recent successes of MHD-based models in accounting for observed pedestal constraints and ELM onset in present devices [13,4,11,12], motivates a detailed study of pedestal MHD stability behaviour in proposed burning plasma tokamak devices. Here, model equilibria based on the superconducting ITER and the compact high field FIRE devices are considered, following a slightly different procedure from that described in sections 2 and 3.

In order to characterize pedestal MHD stability limits, a large number of equilibria must be constructed which incrementally vary the pedestal width and height. For these purposes the details of the equilibrium deep in the core are relatively unimportant, but are broadly consistent with expected profiles for each device.

Model equilibria have been constructed to match the global parameters for each machine given in table 2. These are consistent with the design parameters for the reference cases, except for small modifications to the density of FIRE.

Density and temperature profiles are given a hyperbolic tangent shape in the pedestal (resembling measured profiles (see, e.g. $[14,15]$ ), and a simple polynomial dependence in the core:

$n_{\mathrm{e}}(\Psi)=n_{\text {sep }}+a_{n 0}\left\{\tanh \left[\frac{2\left(1-\Psi_{\text {mid }}\right)}{\Delta}\right]-\tanh \left[\frac{2\left(\Psi-\Psi_{\text {mid }}\right)}{\Delta}\right]\right\}+a_{n 1}\left[1-\left(\frac{\Psi}{\Psi_{\text {ped }}}\right)^{\alpha_{n 1}}\right]^{\alpha_{n 2}}$
$T(\Psi)=T_{\text {sep }}+a_{T 0}\left\{\tanh \left[\frac{2\left(1-\Psi_{\text {mid }}\right)}{\Delta}\right]-\tanh \left[\frac{2\left(\Psi-\Psi_{\text {mid }}\right)}{\Delta}\right]\right\}+a_{T 1}\left[1-\left(\frac{\Psi}{\Psi_{\text {ped }}}\right)^{\alpha_{T 1}}\right]^{\alpha_{T 2}}$

where $\Psi$ is the normalized poloidal flux, and $\Delta$ is the pedestal width in $\Psi$ space. The constants $a_{0}$ and $a_{1}$ are chosen to give the desired pedestal and axis values, and $\alpha_{0}$ and $\alpha_{1}$ are chosen to approximately match expected core profiles from transport codes. For the baseline cases, we select $n_{\text {ped }}=0.71\left\langle n_{\mathrm{e}}\right\rangle, n_{0}=1.1\left\langle n_{\mathrm{e}}\right\rangle, n_{\text {sep }}=0.3\left\langle n_{\mathrm{e}}\right\rangle, \alpha_{n 0}=1, \alpha_{n 1}=0.5, \alpha_{T 0}=1$, and $\alpha_{T 1}=2$.

In the pedestal region, the parallel current is taken to be equal to the bootstrap current, as calculated using a simplified version of the Sauter collisional model [16]. In the core, where details of the current are relatively unimportant, the profile is taken to have a simple polynomial

Table 2. Reference parameters for the model equilibria in section 4.

\begin{tabular}{lll}
\hline & ITER & FIRE \\
\hline$B_{\mathrm{t}}(\mathrm{T})$ & 5.3 & 10 \\
$I_{\mathrm{p}}(\mathrm{MA})$ & 15 & 7.7 \\
$R(\mathrm{~m})$ & 6.2 & 2.14 \\
$a(\mathrm{~m})$ & 2.0 & 0.595 \\
$\kappa_{\mathrm{a}}$ & 1.85 & 2.0 \\
$\delta_{\mathrm{a}}$ & 0.49 & 0.7 \\
$\left\langle n_{\mathrm{e}}\right\rangle\left(10^{20} \mathrm{~m}^{-3}\right)$ & 1.0 & 3.6 \\
\hline
\end{tabular}



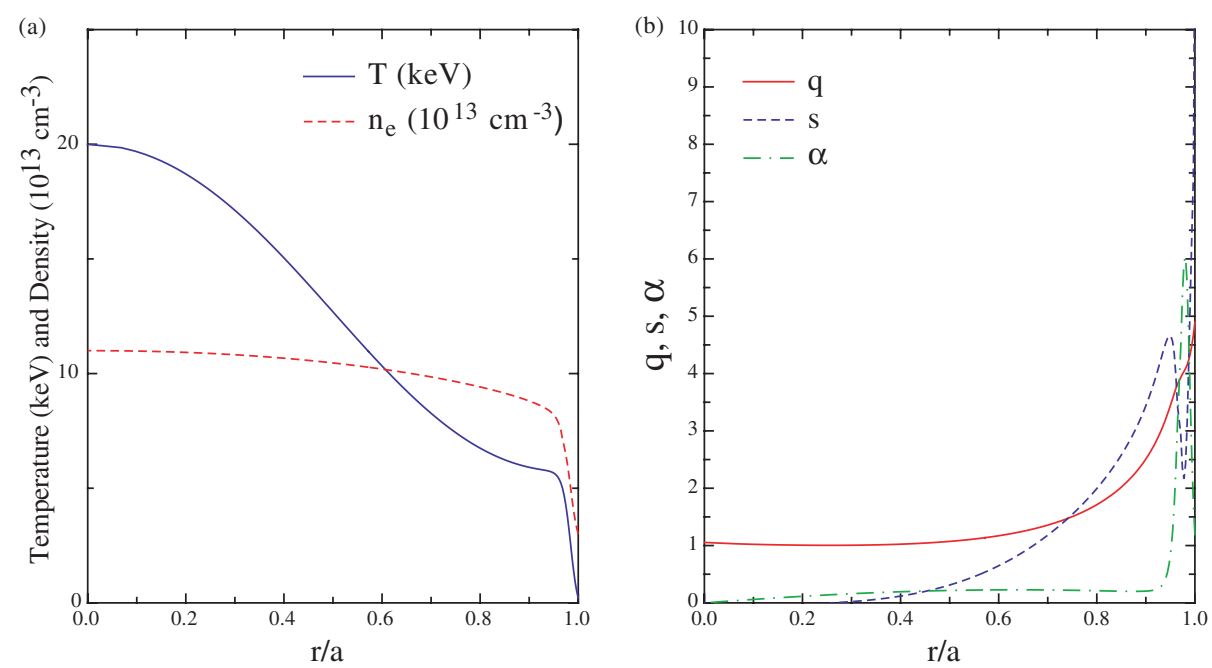

Figure 7. Equilibrium profiles for a sample ITER case with pedestal width of $\Delta / a \sim 0.03$, and pedestal temperature $T_{\text {ped }} \sim 5 \mathrm{keV}$.

form, with coefficients chosen to give a central $q_{0}=1.05$, and a total plasma current $\left(I_{\mathrm{p}}\right)$ as in table 2.

A number of simplifications are made to streamline the equilibrium construction process, including up-down symmetry (while matching the given separatrix elongation and triangularity), and ignoring true X-points. The equilibria are calculated using the TOQ code.

To characterize the pedestal stability constraints, the pedestal width $(\Delta)$ is varied, and at each value of $\Delta$, the pedestal temperature is increased incrementally (with the bootstrap current calculated self-consistently) until stability boundaries are crossed. Sample model ITER equilibrium profiles near the stability boundary at a pedestal width $\Delta / a \sim 0.03$ are shown in figure 7.

The intermediate $n(n>\sim 5)$ MHD code ELITE [6] is again used in the pedestal stability calculations. ELITE has been successfully benchmarked against the GATO [17] and MISHKA [18] codes, and allows efficient calculation of the pedestal stability bounds, and the growth rates and mode structures of the limiting instabilities [6,4]. A sample of toroidal mode numbers, $n=8,10,15,20,30$, are studied, over the range expected to be most unstable. A finite growth rate $(\gamma)$ threshold $\left(\gamma / \omega_{\mathrm{A}}>0.01\right.$, where $\omega_{\mathrm{A}}=B / R(4 \pi \rho)^{-1 / 2}$ with $\rho$ the plasma mass density at the edge) is used as a threshold for 'instability', eliminating slow growing modes unlikely to trigger ELMs.

The results of these calculations of intermediate- $n$ ideal stability bounds on $T_{\text {ped }}$ (at fixed $\left.n_{\text {ped }}\right)$ as a function of the pedestal width are given for each machine in figure 8 . Note that at narrow pedestal widths, relatively high $n$ modes are the most unstable for both cases, while at wider pedestal widths, high $n$ modes become second stable, and modes in the range $n \sim 10-20$ are most unstable. The maximum stable pedestal temperature is found to be a monotonically increasing function of pedestal width, but the dependence is sub-linear, particularly at small widths. In studies using weakly shaped (e.g. low triangularity) equilibria, the decrease in maximum stable pedestal gradient with width is marked: very roughly $\beta_{\text {ped }} \propto \Delta^{2 / 3}$. However, for the strongly shaped ITER and FIRE equilibria, this tendency for the maximum stable gradient to decrease with pedestal width is partially offset by increasing second stability access at higher widths. 

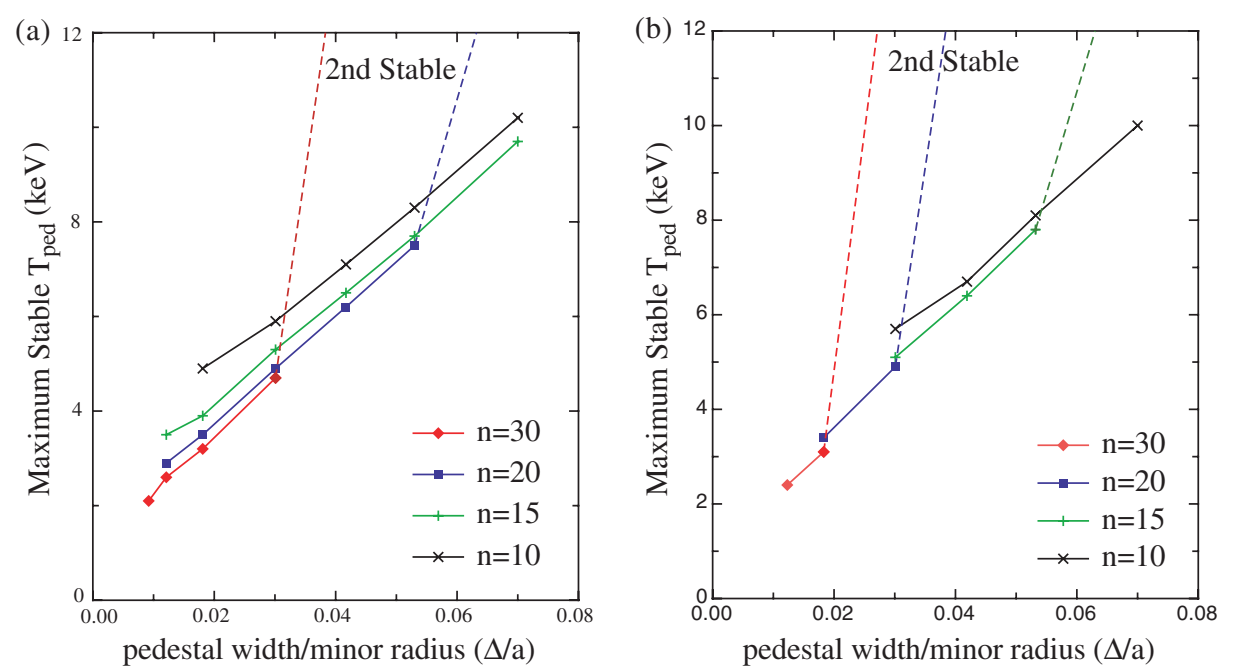

Figure 8. Maximum stable pedestal temperature for model equilibria of each device, as a function of the pedestal width. Stability boundaries for toroidal mode numbers $n=10,15,20,30$ are shown, for $(a)$ ITER with $n_{\text {eped }}=7.1 \times 10^{13} \mathrm{~cm}^{-3}$ and $(b)$ FIRE with $n_{\text {eped }}=2.6 \times 10^{14} \mathrm{~cm}^{-3}$.

Because the ITER and FIRE equilibria have different values of the pedestal density and magnetic field, it is useful to compare the pedestal stability constraints using normalized quantities. Figure 9 shows the pedestal stability constraints imposed by the most unstable of the wavelengths studied, in terms of the normalized pedestal $\beta\left(\beta_{\text {Nped }}=\beta_{\text {ped }} I / a B\right)$, the pedestal $\beta\left(\beta_{\text {ped }}\right)$, and the MHD $\alpha$ parameter at the pedestal centre, where

$$
\alpha \equiv-\frac{2 V^{\prime}}{(2 \pi)^{2}}\left(\frac{V}{2 \pi^{2} R_{0}}\right)^{1 / 2} \mu_{0} p^{\prime}
$$

and $V$ is the plasma volume and a prime represents a derivative with respect to poloidal flux. The apparent trend is that the pedestal beta limits, as a function of $\Delta / a$ are remarkably similar for the two devices. Note that the maximum stable $\alpha$ value is not a constant but, rather, decreases strongly with increasing pedestal width. This is because finite- $n$ modes are sensitive to non-local equilibrium changes across the pedestal and not just the steepest local gradient; at narrow widths the modes extend radially across the entire pedestal region and into the core, hence they are stabilized by decreases in pedestal width at a fixed gradient. Furthermore, the average magnetic shear across the pedestal generally decreases with increasing pedestal width.

\subsection{Variation with triangularity and density}

The pedestal stability results above employ equilibria with the standard set of equilibrium parameters given in table 2. It is also of interest to study how these boundaries change with changes in the equilibria, particularly changes in parameters such as triangularity and density that are expected to be at least partially controllable in experiments.

The triangularity of the plasma cross-section has been found both theoretically and experimentally to have a significant impact on the pedestal, as shown above and in, e.g. [13-15, 19-21]. In particular, higher triangularity generally improves the effective average curvature and increases pedestal beta limits at a given pedestal width. At high triangularity the peeling and ballooning branches of the instability begin to decouple, and second stability access can become possible for high $n$ modes (as illustrated in figure 5, for example). 

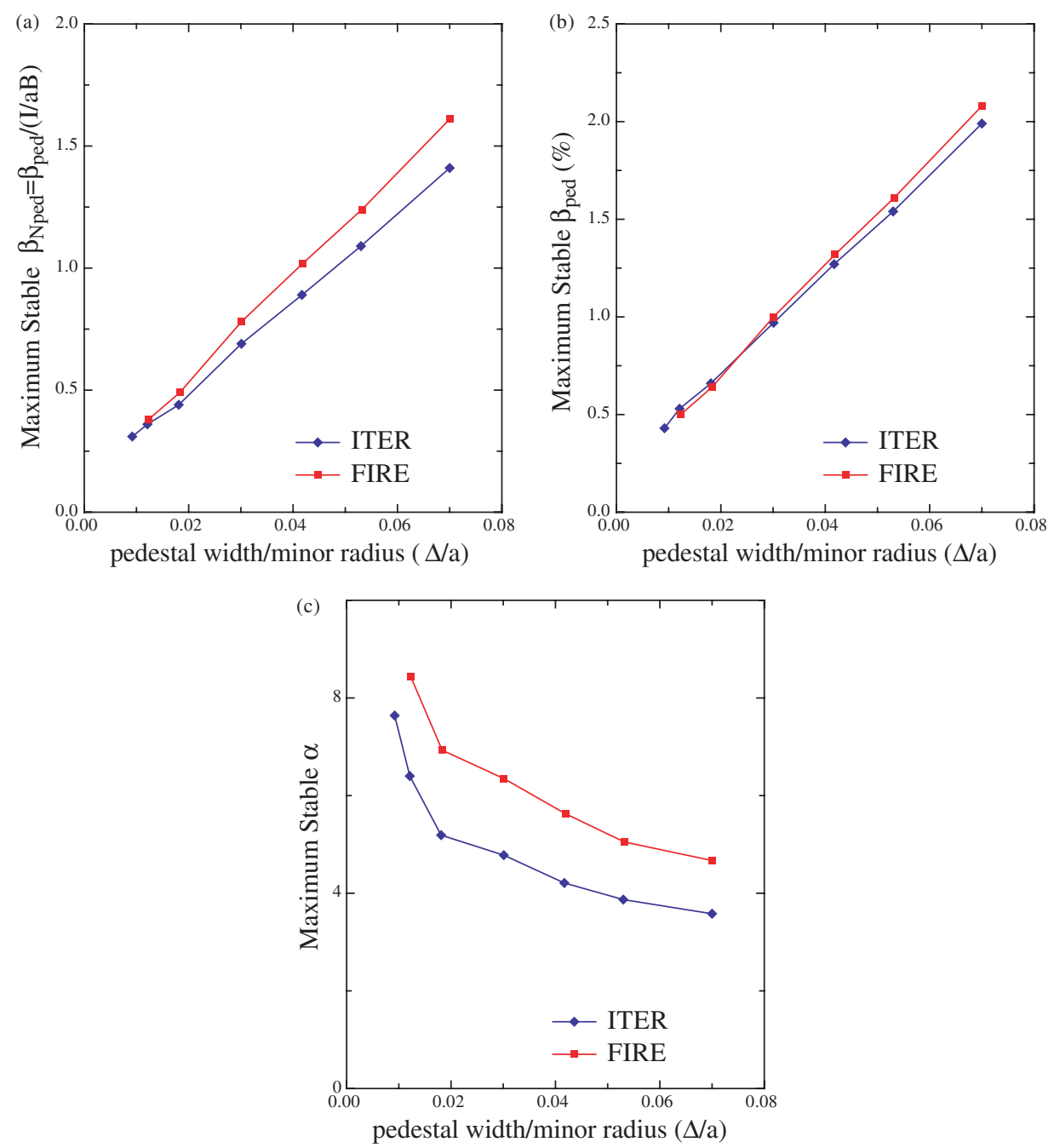

Figure 9. Comparison of pedestal stability limits for model equilibria of the three candidated devices, given in terms of $(a)$ normalized pedestal beta, $(b)$ pedestal beta, and $(c)$ the MHD alpha parameter at the pedestal center; plotted against normalized pedestal width. The stability limit imposed by the most unstable of the studied values of $n(n=8,10,15,20,30)$ is shown.

Figure 10(a) shows the results of a study in which the triangularity of the last closed flux surface $\left(\delta_{\mathrm{a}}\right)$ is varied, while all other parameters in table 2 , and the pedestal width $(5 \%$ of the poloidal flux, or $\Delta / a \sim 0.03$ ) are held fixed. The pedestal stability limits increase roughly a factor of two going from low $(\delta \sim 0)$ to high $(\delta \sim 0.5)$ triangularity and begin to roll over around $\delta \sim 0.5$. However, it should be noted that in these cases, the higher moments of the plasma shape, e.g. the 'squareness', are set to zero. Previous studies suggest that stability limits may continue to increase at very high values of $\delta$ if an optimized squareness is used [22].

Density also can have an impact on pedestal stability limits. Because the current and resulting magnetic shear play an important role in determining pedestal stability limits, these limits have separate dependencies on density and temperature, not just the pressure, as discussed in section 3. Trading off density for temperature at a given pressure alters the 

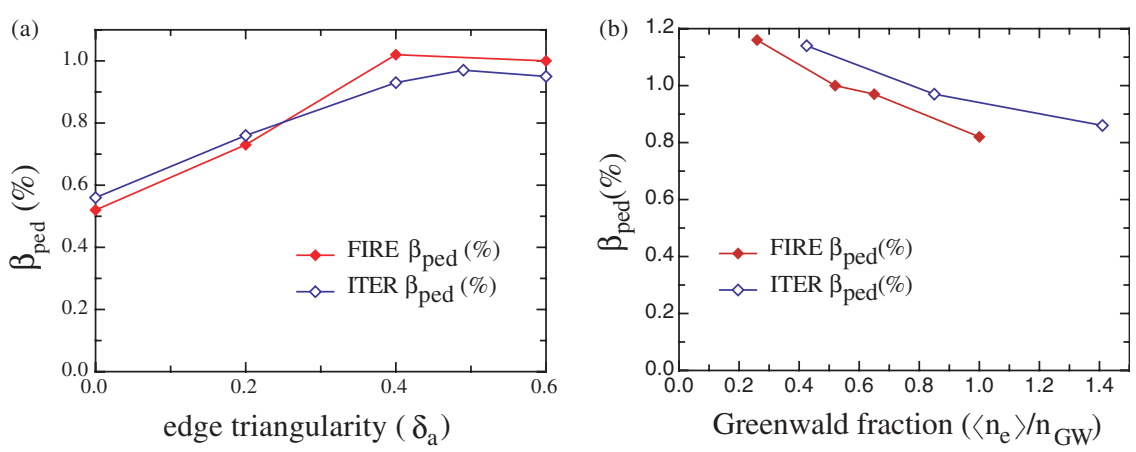

Figure 10. Variation in pedestal stability limits with $(a)$ triangularity and $(b)$ density for FIRE and ITER model equilibria. A fixed pedestal width of $5 \%$ of the poloidal flux $(\Delta / a \sim 0.03)$ is used and all parameters are from table 2 , except the indicated one which is varied.

collisionality and the local value of the bootstrap current. A study of the variation of pedestal stability limits with density is shown in figure $10(b)$. Here, the average density is varied with the pedestal density fixed at 0.71 of the average value, and the separatrix density fixed at 0.3 of the average. Lower density results in increased bootstrap current, lower shear, increased second stability access, and higher stability limits.

\subsection{Unstable mode structure}

The linear mode structure of the most unstable mode is expected to be related to the size of the resulting ELM, though nonlinear dynamics and scrape-off-layer physics are likely to play a significant role (see, e.g. $[23,3,4,11,12,21])$.

The mode structures of the limiting instabilities in the model equilibria for both devices have a characteristic peeling-ballooning mode structure, localized to the outboard midplane, and extending radially somewhat beyond the pedestal. Lower $n$ modes tend to have broader radial extent than higher $n$ modes, though the difference can be relatively small for a given equilibrium. Note however that for these equilibria, lower $n$ modes are most unstable when the pedestal is wide, so there exists a clear correlation between the wavenumber of the most unstable mode and its radial extent (which is wider for wider pedestals). Example mode structures are given in figure 11.

\subsection{Non-ideal MHD effects}

A host of non-ideal-MHD and rotational plasma physics can potentially modify the results of the previous sections, including toroidal flow shear, finite resistivity, finite Larmor radius effects, kinetic resonance effects, and $E \times B$ shear.

Diamagnetic stabilization of short to intermediate wavelength instabilities has been identified as a potentially important piece of non-ideal physics in the pedestal regime (see, e.g. [24-28]). A full treatment of diamagnetic effects requires a detailed kinetic or two-fluid formalism beyond the scope of this study. However, simple models can be used to estimate the impact of diamagnetic stabilization on the results from the previous section. The usual approach is to compare the calculated ideal MHD growth rate to the ion diamagnetic frequency $\left(\omega_{* \mathrm{pi}}\right)$, employing $\gamma_{\mathrm{MHD}}>\omega_{* \mathrm{pi}} / 2$ as the modified threshold for instability in the presence of diamagnetic stabilization $[25,26]$. This approach requires selecting a characteristic local value 

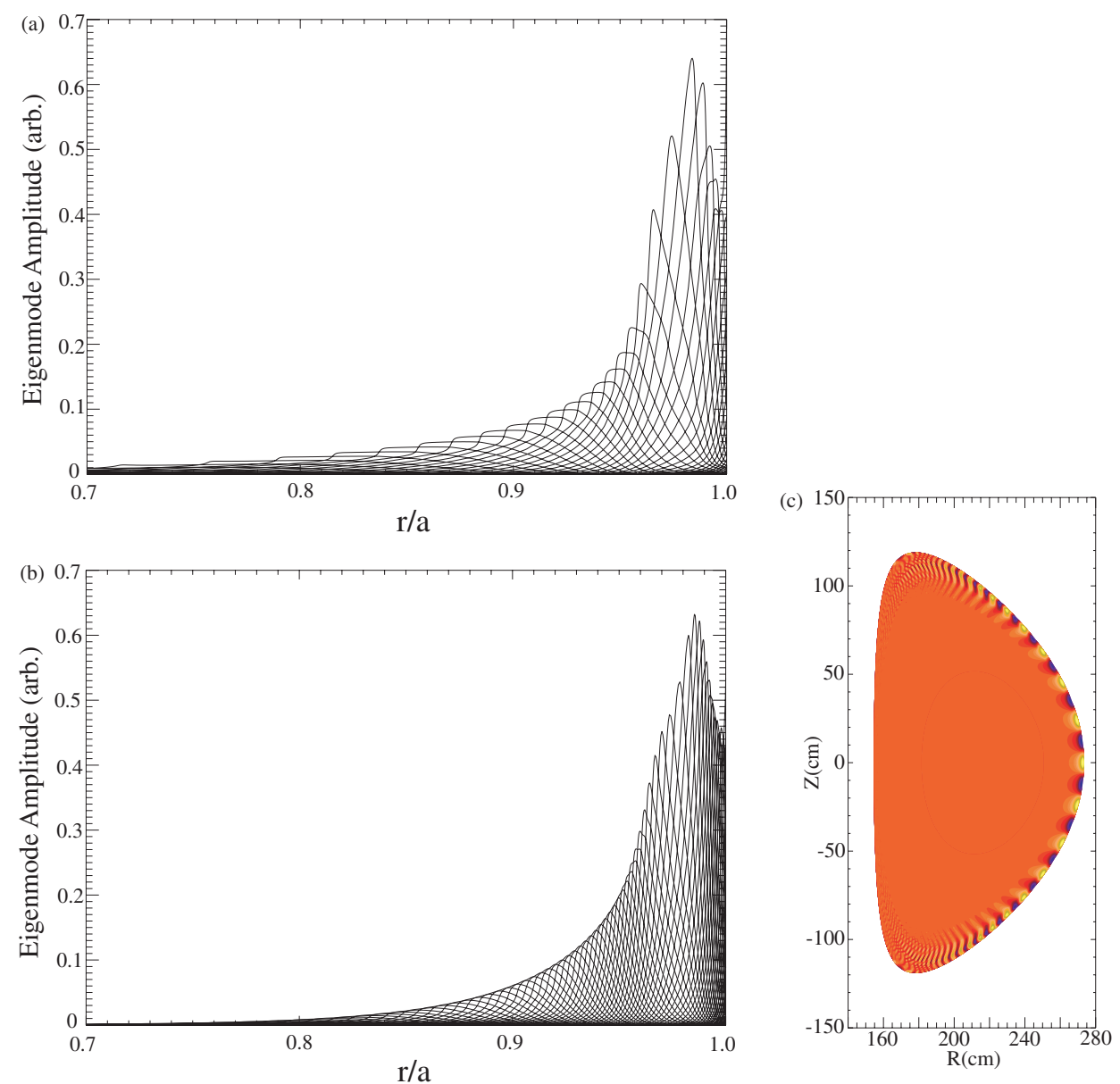

Figure 11. Comparison of radial eigenmode structures for $(a) n=8$, (b) $n=20$ in model ITER equilibrium with $\Delta / a=0.03, T_{\text {ped }}=6.2 \mathrm{keV}$. $(c)$ two-dimensional structure of an $n=20$ peeling-ballooning mode in model FIRE equilibrium with $\Delta / a=0.03, T_{\text {ped }}=5 \mathrm{keV}$.

of $\omega_{* \mathrm{pi}}$, a quantity which varies rapidly over the pedestal. Here, we use the maximum value of

$$
\omega_{* \mathrm{pi}} \equiv \frac{c n}{e n_{\mathrm{i}}} \frac{\partial p_{\mathrm{i}}}{\partial \psi}
$$

in the pedestal region divided by $\sqrt{2}$ (such that the square of $\omega_{* \mathrm{pi}}$ takes on its half maximum value). Results obtained using this simple local model of diamagnetic stabilization are shown in figure 12. (Note that values of $\gamma_{\text {MHD }}$ used here are from a compressionless model. These results are approximate and are intended only to provide an indication of the impact of diamagnetic effects.) One limitation of this local model is that it assumes a constant level of diamagnetic stabilization over the full radial extent of the mode, which can extend beyond the pedestal. For narrow pedestals this can lead to a significant overestimate of the stabilizing effect. A modification suggested by Rogers and Drake $[29]^{3}$ allows a smooth transition from the regime where the pedestal is much wider than the mode to the regime in which it is

3 A similar model is employed in the context of a circular s-alpha model in [27]. 

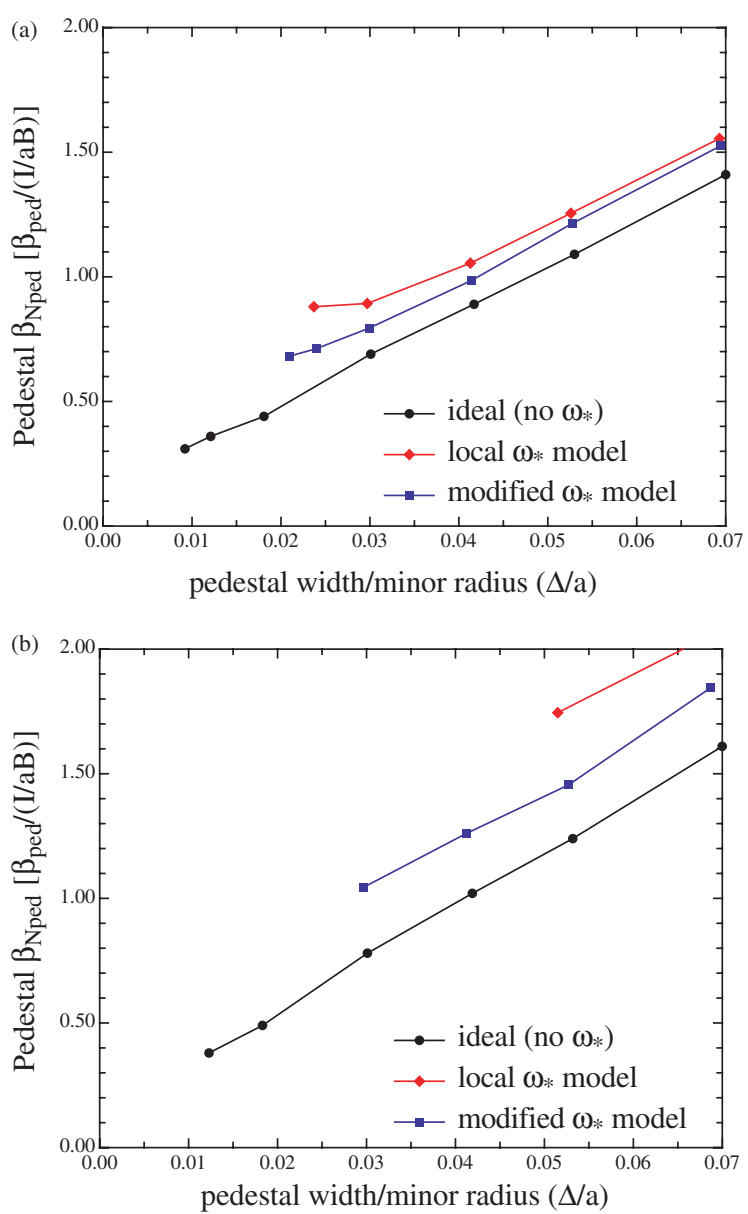

Figure 12. Stability constraints (from $n=8,10,15,20,30$ modes) on the normalized pedestal beta as a function of pedestal width for $(a)$ ITER and $(b)$ FIRE, including results with the local $\gamma_{\text {MHD }}>\omega_{* \text { pi }} / 2$ model of diamagnetic stabilization, and with the modified model described in the text.

much narrower than the mode. The $\omega_{* \mathrm{pi}} / 2$ term given above is multiplied by a factor of $1 /\left(1+1 / k_{\theta} L_{\mathrm{p}}\right)$. Here, $k_{\theta}$ is the poloidal wave number determined on the outer midplane, and the pressure gradient scale length $\left(L_{\mathrm{p}}\right)$ is taken to be approximately the pedestal half width. Results with this modified diamagnetic stabilization model are also given in figure 12. (Stability limits at narrow widths in the presence of the diamagnetic stabilization are not presented in the figure, but this should not be taken as an indication that no stability limit exists for these cases.) Note that the diamagnetic stabilization at a given pedestal width is larger for FIRE due to its larger value of $\rho / a$, where $\rho$ is the ion gyroradius. It is expected that the simple model presented in figure 12 may overstate the impact of diamagnetic stabilization, due for example to the effect of variation in $\omega_{* \mathrm{pi}}$ across the pedestal [28].

\section{Summary}

Ideal MHD constraints on the H-mode temperature pedestal are explored, and quantified in sets of model equilibria based on the ITER and FIRE designs. Two basic instabilities 
govern ideal MHD in the plasma edge region. First, the high toroidal mode number, $n$, ballooning mode is driven by edge pressure gradient, and therefore imposes a direct limit on the temperature gradient that can be maintained in the transport barrier. On the other hand, for shaped plasmas, sufficient current density can stabilize the ballooning mode, providing access to higher pressure gradients: so-called 'second stability' regime. The other instability is the peeling mode, which is strongly related to the kink mode, but is not necessarily restricted to low $n$. The peeling mode is driven by the edge current density; in contrast to the ballooning mode, the peeling mode is stabilized by pressure gradient (i.e. a larger edge current density can be tolerated at higher pressure gradient). A final twist to the story is that at high pressure gradient and edge current density the ballooning and peeling modes can couple, leading to particularly dangerous modes driven by both current density and pressure gradient, with a relatively large radial extent, typically extending right across the transport barrier region. These 'coupled peeling-ballooning' modes have been explored as a possible cause of large ELMs $[3,4,13]$, and are found in many cases to provide the relevant ideal MHD constraint on the pedestal.

Ideal MHD constraints on the pedestal are calculated as a function of normalized edge current and pressure gradient using series of model equilibria. Because the edge current is generally not well diagnosed in tokamaks, and because it is the pedestal temperature which is needed by core transport models to predict machine performance, it is useful to recast the stability diagrams in terms of pedestal temperature and pressure gradient. A key observation of this paper is that, because higher temperature leads to lower collisionality (increasing bootstrap current) and lower resistivity (increasing Ohmic current), there is generally a monotonic relationship between pedestal temperature and current. Thus, in the limit of a steady state current, it is possible to calculate directly MHD constraints on the pedestal temperature itself. By constructing multiple series of model equilibria, it is then possible to calculate these pedestal temperature constraints as a function of relevant parameters such as discharge shape, pedestal width, and pedestal density. In general, the current in the edge region during the ELMing phase would be somewhat lower than the steady state predictions adopted in our study (assuming that the ELMs expel edge current), suggesting that, in some cases, somewhat higher pressure pedestals than those quoted here could be accommodated. Indeed we note that the expected slow evolution of the current profile has inspired qualitative models of various types of dynamic ELM cycle, for example those in [3,4]. To quantify this would require a self-consistent transport analysis, which we recommend for future work, but is not attempted here.

A set of model equilibria with varying pedestal width $(\Delta)$ and height have been constructed for the ITER and FIRE devices, and constraints imposed by intermediate $n$ MHD modes have been assessed using the ELITE code. The calculated stability bounds on $\beta_{\text {ped }}$ vs $\Delta / a$ are similar between the machines. The maximum stable pedestal height $\left(\beta_{\text {ped }}\right)$ is a strong function of the width, though notably sub-linear particularly at narrow widths. The maximum stable height is a strong function of triangularity, and a weaker function of density. Intermediate to high $n$ modes are found to be the limiting instabilities, and calculated mode structures, which are expected to be related to the ELM depth, extend inward somewhat beyond the pedestal. Diamagnetic effects on the stability bounds are assessed with a local model (i.e. instability for $\gamma>\omega_{* \mathrm{pi}} / 2$ ), and with a modified local model which takes into account the finite ratio of mode width to pedestal width. Diamagnetic effects, as modelled by these simple prescriptions, are found to significantly increase the ideal MHD bounds and to shift the limiting mode to longer wavelengths, though it is expected that the simple models may overestimate the diamagnetic stabilization. It is possible to combine the calculated pedestal temperature constraints from figure $9(a)$ with core transport studies such as those in [1], to give performance projections. 
These projections will be a strong function of the pedestal width, and the physics determining this width remains a key uncertainty.

In conclusion, we have developed a procedure for evaluating ideal MHD constraints on the temperature pedestal, and have applied this technique to characterize these constraints as a function of shape, pedestal width and pedestal density for model ITER and FIRE equilibria. Ideal MHD calculations of pedestal constraints have proved to be useful for understanding pedestal and ELM observations on present machines, and provide useful guidelines for projecting performance of future devices. A complete, fully predictive understanding of the pedestal height likely awaits advances in dynamical modelling, including transport processes and accounting for non-ideal effects, as well as understanding of the physics governing the pedestal width.

\section{Acknowledgments}

The authors gratefully acknowledge contributions from RL Miller, JW Connor, B Rogers and J Drake. This work was funded by the US Department of Energy under Grant No DE-FG0395ER54309, the United Kingdom Engineering and Physical Sciences Research Council and Euratom.

\section{References}

[1] Kinsey J E, Onjun T, Bateman G, Kritz A, Pankin A, Staebler G and Waltz R E 2002 Burning plasma projections using drift wave transport models and scalings for the H-mode pedestal Proc. 19th IAEA Fusion Energy Conf. (Lyon, France, 2002) Nucl. Fusion (Vienna: IAEA) paper IAEA-CN-94/TH/P1-09, submitted

[2] Lortz D 1975 Nucl. Fusion 1549

[3] Connor J W, Hastie R J, Wilson H R and Miller R L 1998 Phys. Plasmas 52687

Hegna C C, Connor J W, Hastie R J and Wilson H R 1996 Phys. Plasmas 3584

Wilson H R and Miller R L 1999 Phys. Plasmas 6873

[4] Snyder P B et al 2002 Phys. Plasmas 92037

[5] Bickerton R J, Connor J W and Taylor J B 1971 Nature (London) Phys. Sci. 229110

[6] Wilson H R, Snyder P B, Huysmans G T A and Miller R L 2002 Phys. Plasmas 91277

[7] Wilson H R 1994 UKAEA Fusion Rep. UKAEA FUS 271

[8] Hirshman S P and Sigmar D J 1981 Nucl. Fusion 211079

[9] Hirshman S P, Hawryluk R J and Birge B 1977 Nucl. Fusion 17611

[10] Connor J W, Hastie R J and Taylor J B 1979 Proc. R. Soc. Series A 3651

[11] Saarelma S, Gunter S, Kurki-Suonio T and Zehrfeld H-P 2000 Plasma Phys. Control. Fusion 42 A139

[12] Mossessian D et al 2002 Plasma Phys. Control. Fusion 44 423-37

[13] Snyder P B et al 2002 IAEA Fusion Energy Conf. (Lyon, France) Nucl. Fusion. submitted

[14] Osborne T H et al 2000 Plasma Phys. Control. Fusion 42 A175

[15] Hubbard A E 2000 Plasma Phys. Control. Fusion 42 A15

[16] Sauter O, Angioni C and Lin-Liu Y R 1999 Phys. Plasmas 62834

[17] Bernard L C et al 1981 Comp. Phys. Commun. 24377

[18] Mikailovskii A B, Huysmans G T A, Sharapov S E and Kerner W 1997 Plasma Phys. Rep. 23844

[19] Hatae T, Sugihara M and Hubbard A E 2001 Nucl. Fusion 41285

[20] Huysmans G T A et al 1999 Nucl. Fusion 391489 Becoulet M et al 2002 Plasma Phys. Control. Fusion 44 A103

[21] Ferron J R et al 2000 Phys. Plasmas 71976 Strait E J et al 1993 Proc. 20th EPS Conf. on Plasma Physics and Controlled Fusion Research (Lisbon, Portugal) Ferron J R et al 1995 Proc. 22nd EPS Conf. on Plasma Physics and Controlled Fusion Research Osborne T H et al 1997 Proc. 24th EPS Conf. Plasma Phys. Control. Fusion

[22] Turnbull A D et al 1999 Phys. Plasmas 61113 Turnbull A D 2002 Private Communication

[23] Lao L L 2000 Plasma Phys. Control. Fusion 42 A51

[24] Roberts K V and Taylor J B 1962 Phys. Rev. Lett. 8197 
[25] Tang W M, Dewar R L and Manickam J 1982 Nucl. Fusion 221079

[26] Hahm T S and Diamond P H 1987 Phys. Fluids 30133

[27] Rogers B N and Drake J F 1999 Phys. Plasmas 62797

[28] Hastie R J, Catto P J and Ramos J J 2000 Phys. Plasmas 74561

[29] Rogers B N and Drake J F 2002 Private communication 